\title{
SELF-RECOVERING SECTION OF RPV STEEL RADIATION EMBRITTLEMENT KINETICS AS INDICATION OF MATERIAL INTELLIGENT BEHAVIOUR
}

\section{EVGENII KRASIKOV}

National Research Centre

Kurchatov Institute

Kurchatov Sq., 1

Moscow 123182

Russia

e-mail: ekrasikov@mail.ru

\begin{abstract}
Influence of neutron irradiation on reactor pressure vessel (RPV) steel degradation are examined with reference to the possible reasons of the substantial experimental data scatter and furthermore - nonstandard (nonmonotonous) and oscillatory embrittlement behaviour. In our glance, this phenomenon may be explained by presence of the wavelike recovering component in the embrittlement kinetics.

We suppose that the main factor affecting steel anomalous embrittlement is fast neutron intensity (dose rate or flux), flux effect manifestation depends on stateof-the-art fluence level. At low fluencies radiation degradation has to exceed normative value, then approaches to normative meaning and finally became sub normative. Data on radiation damage change including through the ex-service RPVs taking into account chemical factor, fast neutron fluence and neutron flux were obtained and analyzed.
\end{abstract}

Keywords and phrases: reactor pressure vessel, steel, self-recovering section, embrittlement kinetics, material intelligent behaviour.

Received November 30, 2015

(ㄷ) 2016 Scientific Advances Publishers 
In our opinion controversy in the estimation on neutron flux on radiation degradation impact may be explained by presence of the wavelike component in the embrittlement kinetics. Therefore, flux effect manifestation depends on fluence level. At low fluencies radiation degradation has to exceed normative value, then approaches to normative meaning and finally became sub normative. As a result of dose rate effect manifestation peripheral RPV's zones in some range of fluencies have to be damaged to a large extent than situated closely to core.

Moreover as a hypothesis, we suppose that at some stages of irradiation damaged metal have to be partially restored by irradiation, i.e., neutron bombardment. Nascent during irradiation structure undergo occurring once or periodically transformation in a direction both degradation and recovery of the initial properties. According to our hypothesis at some stage(s) of metal structure degradation neutron bombardment became recovering factor. Selfrecovering section of RPV steel radiation embrittlement kinetics as indication of material intelligent behaviour. As a result oscillation arise that in tern lead to enhanced data scatter. In this case, we have to consider irradiation as a recovery factor.

For the sake of correctness, it is necessary to remember that there is an example when contrary to the famous radiation embrittlement in metals neutron irradiation at some range of fast neutron doses is in position to improve both the strength and ductility of steel.

Foregoing hypothetical assumptions on "low-dose effects" in terms "radiation embrittlement contains oscillatory component" and "radiation annealing of the radiation embrittlement" is questionable and needs additional experimental verification and profound scientific study.

\section{Introduction}

Operating PWR's reactor pressure vessels are subject to multi-factor influence. It is practically impossible to reproduce some of this factors (" long-time bias", e.g.) in the framework of experimental investigations including RPV surveillance specimens tests. Detailed information that can be obtained by means of taking through RPV wall samples immediate from the decommissioned RPVs is more representative than received by any another ways and therefore has a highest value.

Testing of the specimens presented a unique opportunity for qualifying consequences of long term irradiation, multi-factor influence and dose rate (flux) effect on actual RPV properties. Data on radiation 
damage change through the ex-service RPV walls taking into account chemical factor and neutron flux were obtained.

Controversy in the opinions on neutron flux on radiation degradation impact may be explained by presence of the wavelike component in the embrittlement kinetics that in turn is an indication of material intelligent behaviour at some sections of RPV steel radiation embrittlement kinetics.

\section{Experimental Procedures}

Along with routine investigations in Russia, systematic research on actual radiation embrittlement of the decommissioned PWR pressure vessel via through samples (trepans) has been carried out.

The earliest commercial PWR prototype unit Novovoronezh-1 (NV1) RPV after 20 years of operation was trepanned in 1987. Then Novovoronezh-2 (NV2), the oldest PWR type experimental reactorprototype ERP and nuclear icebreaker (NIB) "Lenin " RPVs also were trepanned. The most interesting and unexpected data were discovered during trepans of the first nuclear ship - icebreaker "Lenin " investigation.

Chemical analyses of the icebreaker RPV material were carried out with FSQ « Baird » optical emission spectrometer. Results can be seen in Table 1.

Table 1. Chemical composition of the icebreaker RPV materials under study [\%wt.]

\begin{tabular}{|c|c|c|c|c|c|c|c|c|c|}
\hline Material & $\mathrm{C}$ & $\mathrm{Mn}$ & $\mathrm{Si}$ & $\mathrm{P}$ & $\mathrm{Cu}$ & $\mathrm{Mo}$ & $\mathrm{Ni}$ & $\mathrm{Cr}$ & $\mathrm{V}$ \\
\hline Weld & 0,05 & 1,03 & 0,41 & 0,035 & 0,15 & 0,49 & 0,17 & 1,39 & 0,15 \\
\hline Base & 0,17 & 0,45 & 0,28 & 0,018 & 0,09 & 0,67 & 0,35 & 2,75 & 0,09 \\
\hline
\end{tabular}


Fast $(\mathrm{E} \geq 0,5 \mathrm{MeV})$ neutron fluence evaluation was based on the specific Mn-54 and Nb-93m activities of the vessels steel and on the $\mathrm{Nb}-93 \mathrm{~m}$ activity of the RPV cladding.

The RPV materials radiation degradation (embrittlement) was determined by finding the ductile-to-brittle transition temperature shift (TTS). RKP-300 impact pendulum test machine for standard Charpy specimens testing was used.

The general dependence of the TTS on radiation embrittlement coefficient (REC) $A_{F}$ is as follows:

$$
\mathrm{TTS}=\mathrm{A}_{\mathrm{F}} \times \mathrm{F}^{\mathrm{n}}
$$

where $A_{F}$ is the radiation embrittlement coefficient and $F$ is the fast $(\mathrm{E} \geq 0,5 \mathrm{MeV})$ neutron fluence in units of $10^{18} \mathrm{~cm}^{-2}$ (fluence factor), $\mathrm{n}-$ coefficient $(\sim 1 / 3)$.

For Russian RPV Cr-Mo-V base steel $\mathrm{A}_{\mathrm{F}}=\mathrm{A} \times(\mathrm{P}+0,07 \mathrm{Cu})$, where $\mathrm{A}_{\mathrm{F}}$ - coefficient $\left(800\right.$ at $\left.270^{\circ} \mathrm{C}\right), \mathrm{P}$ and $\mathrm{Cu}$ - are the weight concentrations of these elements.

\section{Test Results}

The unexpected results of the icebreaker RPV weld and base metal studying are given in Figure 1, where one can recognize that the actual (measured) radiation embrittlement coefficients of the trepan materials for the periphery (remote) zone of the vessel are significantly higher that for the inner part. One can see also that hardness measurement and Charpy impact testing results agree. 


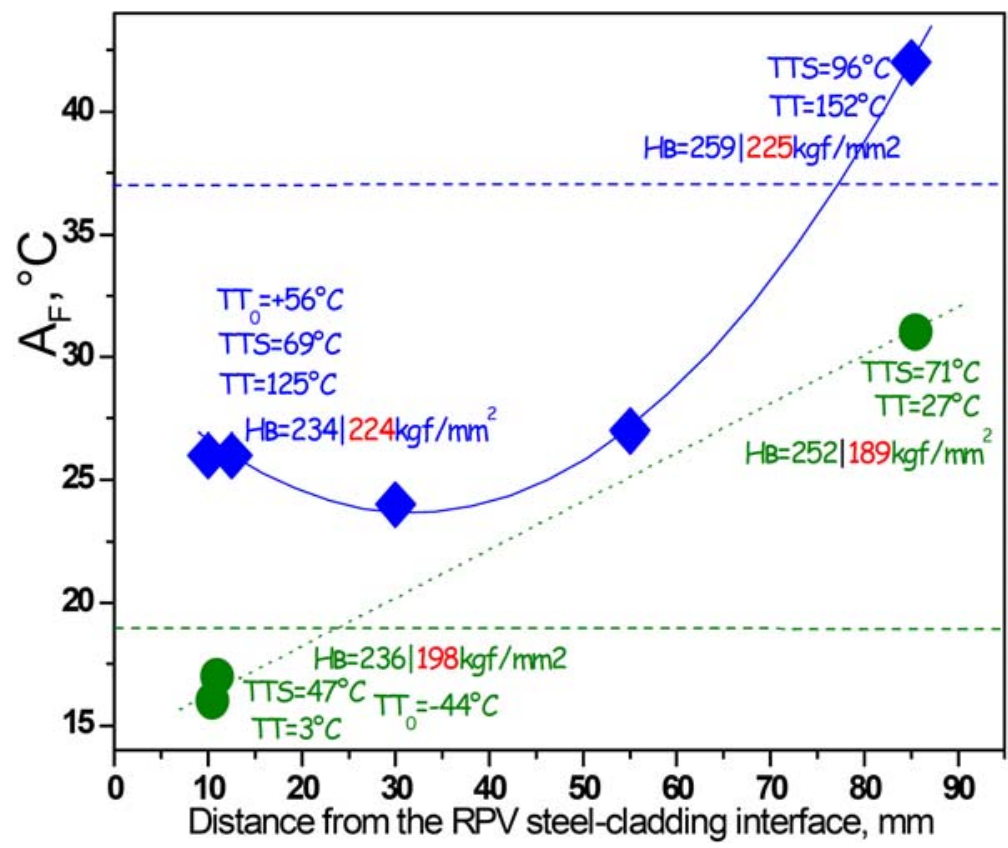

Figure 1. Radiation embrittlement coefficient $A_{F}$ and hardness value HB through the icebreaker RPV wall distribution. TT - transition temperature, $\mathrm{TT}_{0}$ - transition temperature in initial state.

Note: $\mathrm{H}_{\mathrm{B}}=252 \mid 189 \mathrm{kgf} / \mathrm{mm}^{2}$ - hardness values at as-received condition | after annealing $650^{\circ} \mathrm{C} / 2 \mathrm{~h}$.

\section{Data Analysis and Discussion}

First impression from foregoing decommissioned PWR pressure vessel material properties study - enhanced degradation rate at low neutron fluxes. Examples for icebreaker base metal and weld metal are demonstrated in Figures 2 and 3. Registered facts denote that known as "flux effect " factor was in action. Unexpected circumstance however is reduced embrittlement zone appearance that follows after of previous part of enhanced embrittlement. It is seen by means of TTS on neutron flux and fluence dependencies observation. 


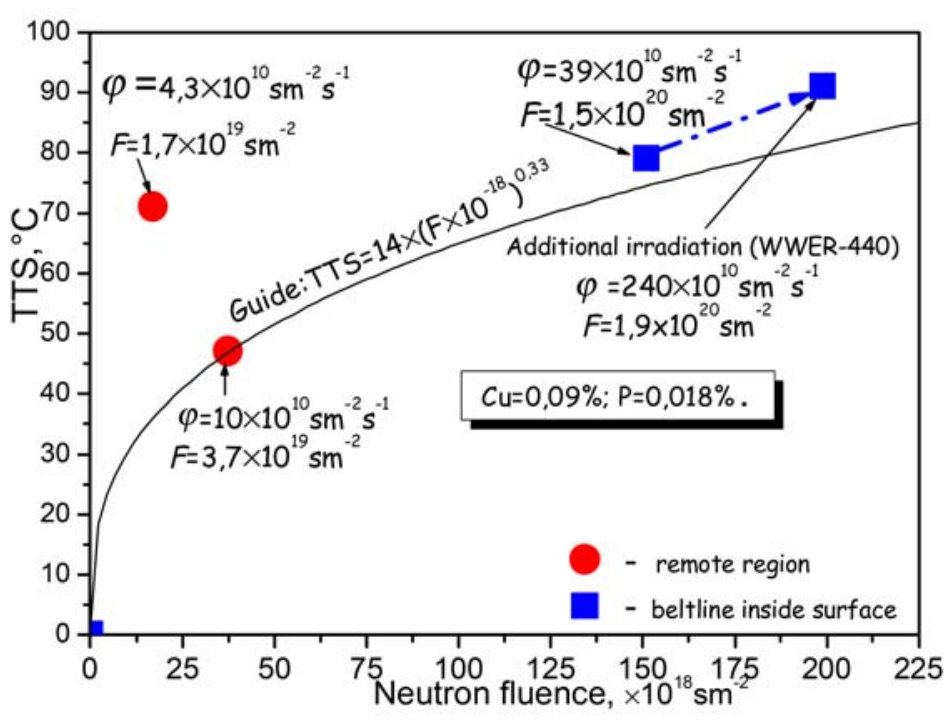

Figure 2. Comparison of the TTSs between "remote " and " inside" icebreaker RPV base metal specimens.

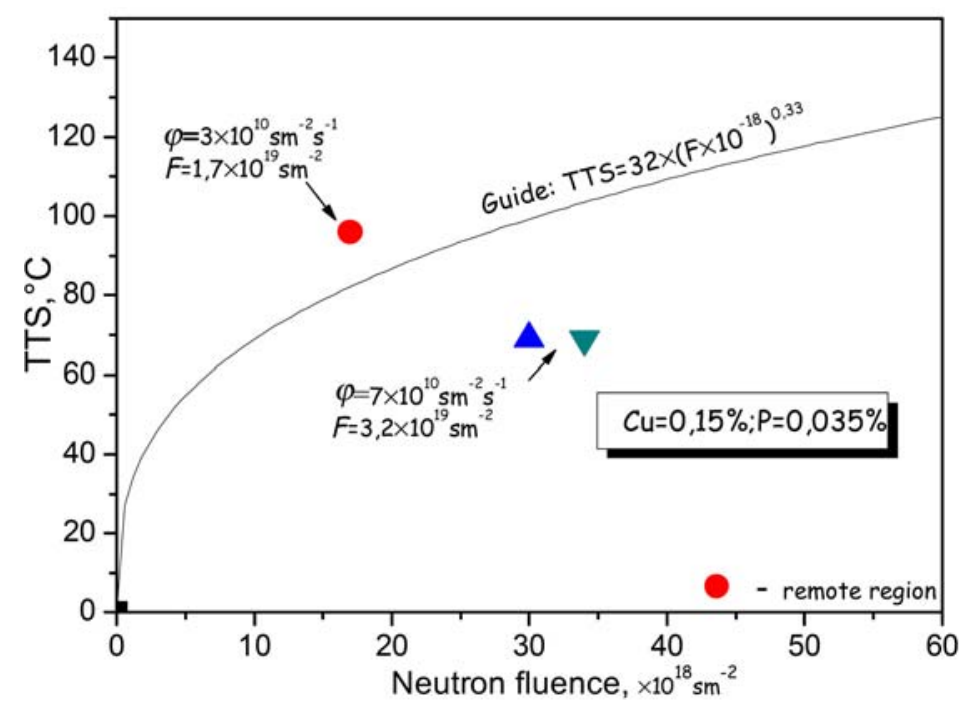

Figure 3. Comparison of the TTSs between " remote " and " inside " icebreaker RPV weld metal specimens. 
In support of the unusual materials gained, we started " flux effect " purposeful study using surveillance specimens from WWER-440/213 RPV. Taking into account, the flux level that is the irradiation position of the selected standard Charpy-type surveillance specimens additional sub size specimens were manufactured and tested. Analysis shows that 3 fold difference in flux level may lead to evident distinction in terms of TTS: $\sim 60^{\circ} \mathrm{C}$ at neutron fluence of $4 \times 10^{19} \mathrm{~cm}^{-2}$ and copper concentration as low as $\sim 0,1 \%$ mass [1].

Search of similar far from trivial effect brings to example where it characterized by authors as « quite atypical » for Doel I, II weld metal SS data (Figure 4 [2]). One can recognize that relatively to Regulatory Guide 1.99 curves zones with opposite effects take place, enlarged shift changes by depressed one.

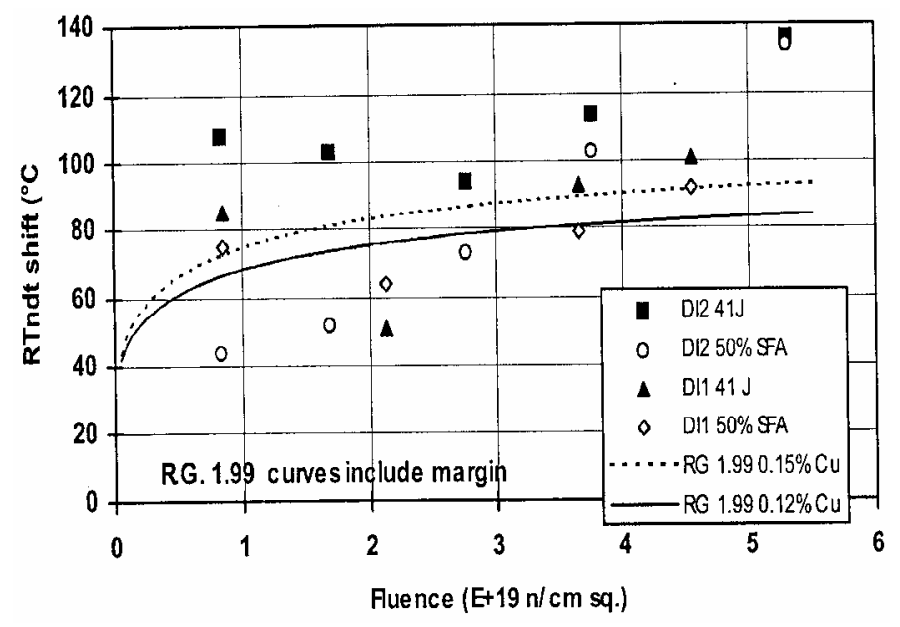

Figure 4. Doel I, II weld - surveillance results.

For the sake of correctness, it is necessary to underline that the first mention concerning distinction between test reactors and low-lead-factor (surveillance) data had appeared as early as 1980 [3].

Summing the previously mentioned on the subject discussed one might conclude, that depending of the fluence level reached manifestation of the "flux effect " in reference to guide pattern may be quite different, namely: negative, positive and " two zeros " as 
represented in Figure 5. Situation looks like famous parable « blind men and an elephant", where a group of blind men (or men in the dark) touch an elephant to learn what it is like. The story is used to indicate that reality may be viewed differently depending upon one's perspective, suggesting that what seems an absolute truth may be relative due to the deceptive nature of half-truths.
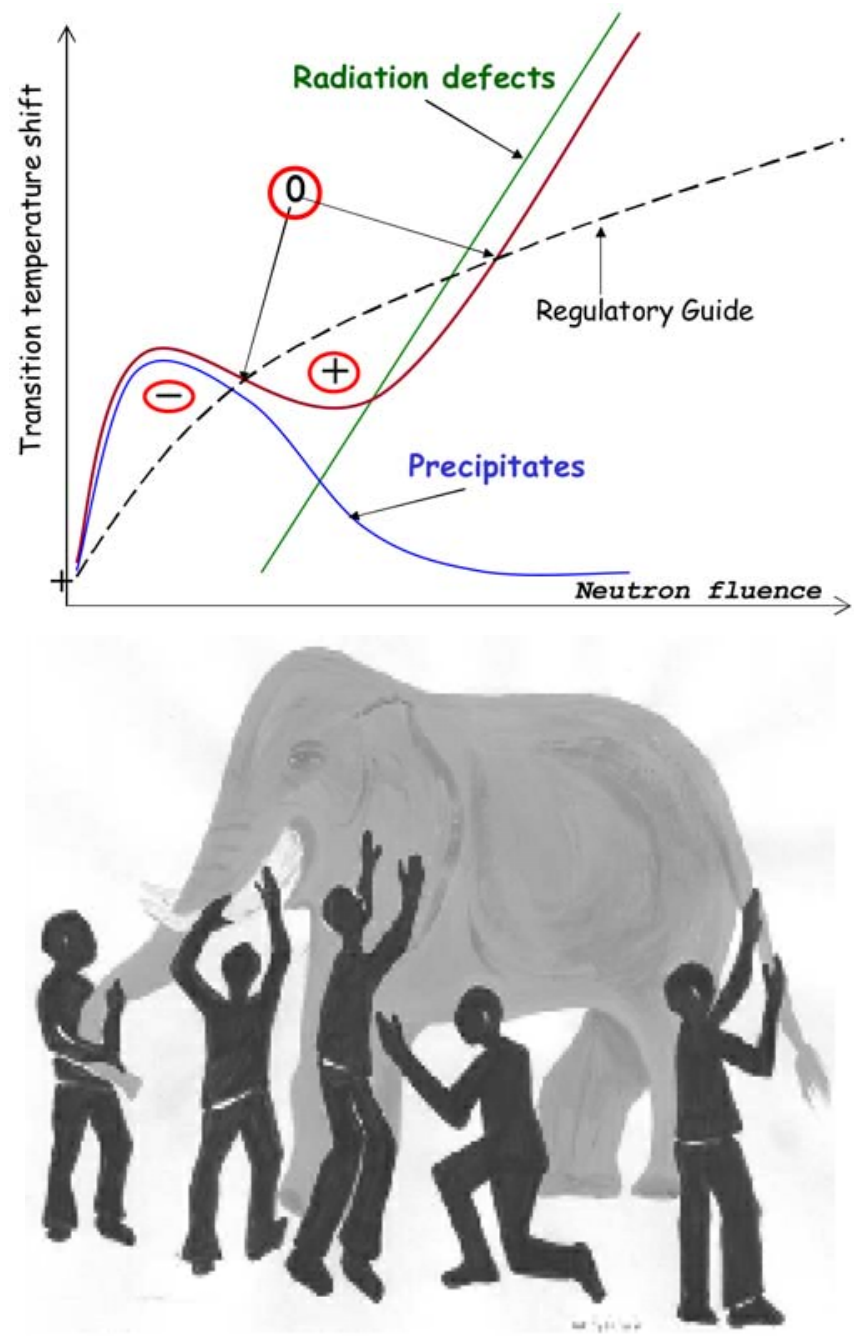

Blind men and an elephant

Figure 5. Sketch of the flux effect manifestation. 
Regulatory guide dependence (formula) has appeared as a result of forced irradiation in test reactors. One can recognize however that specific properties of metal and actual reactor environment may deform this ideal trajectory to the extend that curve monotony character damage. The very wonderful fact is the enhanced degradation aftereffect of the temporary weakening of the embrittlement appearance. Possible comprehensible explanation is as follows: the radiation-induced copperrich precipitates nature (dimensions and concentration) alteration. Evidently, we have fixed phenomenon similar to observed in [4] where neutron irradiation in some range of doses improves the mechanical properties of the unirradiated mild steel (Figure 6).

It is seen that irradiation of unirradiated (initial condition) steel up to dose of $\sim 2,0 \times 10^{18} \mathrm{~cm}^{-2}$ along with strengthening lead to more than 2 -fold ductility increase.

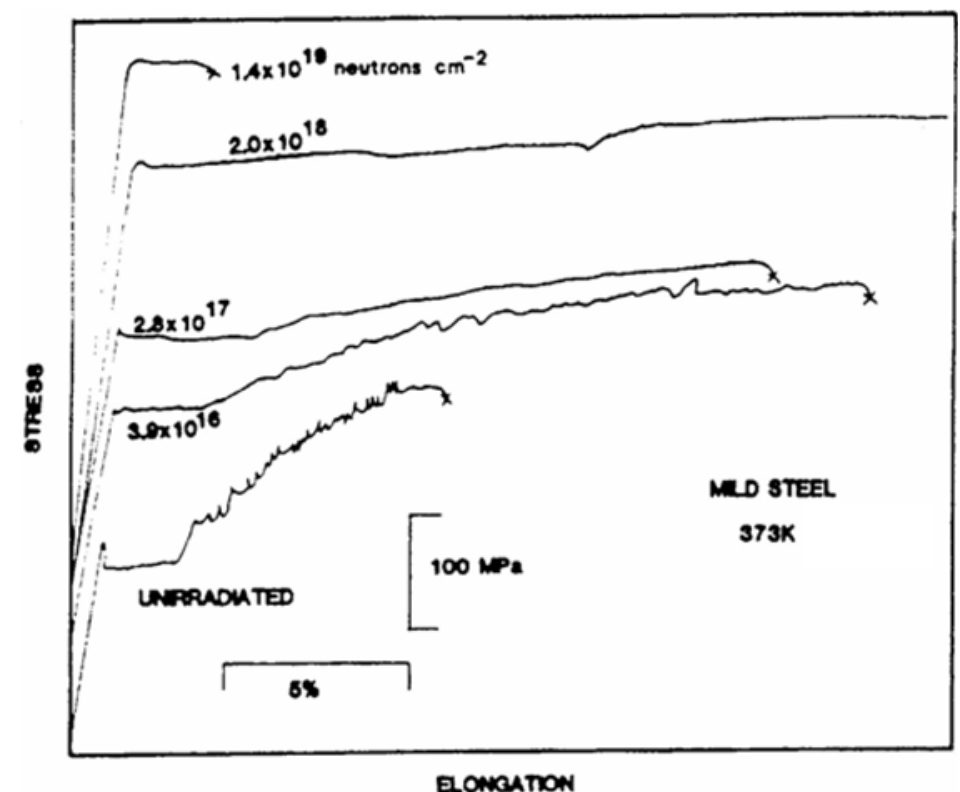

Figure 6. Stress-strain curves of steel as a function of neutron fluence. 
In accordance with sketch of Figure 5, flux effects manifestation depends on fluence level. At low fluencies radiation degradation has to exceed normative value, then approaches to normative meaning and finally became sub normative. As a result of dose rate effect manifestation peripheral RPV's zones in some range of fluencies have to be damaged to a large extent than situated closely to core.

Influence of neutron irradiation on reactor pressure vessel (RPV) steel degradation are examined with reference to the possible reasons of the substantial experimental data scatter and furthermore nonstandard (non-monotonous) and oscillatory embrittlement behaviour. In our glance, this phenomenon may be explained by presence of the wavelike recovering component in the embrittlement kinetics.

We suppose that the main factor affecting steel anomalous embrittlement is fast neutron intensity (dose rate or flux), flux effect manifestation depends on state-of-the-art fluence level. At low fluencies radiation degradation has to exceed normative value, then approaches to normative meaning and finally became sub normative. Data on radiation damage change including through the ex-service RPVs taking into account chemical factor, fast neutron fluence and neutron flux were obtained and analyzed.

In our opinion controversy in the estimation on neutron flux on radiation degradation impact may be explained by presence of the wavelike component in the embrittlement kinetics. Therefore, flux effect manifestation depends on fluence level. At low fluencies radiation degradation has to exceed normative value, then approaches to normative meaning and finally became sub normative.

As a result of dose rate effect manifestation peripheral RPV's zones in some range of fluencies have to be damaged to a large extent than situated closely to core. This finding recently was confirmed by German scientists [5]. 
Moreover as a hypothesis, we suppose that at some stages of irradiation damaged metal have to be partially restored by irradiation, i.e., neutron bombardment. Nascent during irradiation structure undergo occurring once or periodically transformation in a direction both degradation and recovery of the initial properties. According to our hypothesis at some stage(s) of metal structure degradation neutron bombardment became recovering factor. Self-recovering section of RPV steel radiation embrittlement kinetics as indication of material intelligent behaviour. As a result oscillation arise that in tern lead to enhanced data scatter. In this case, we have to consider irradiation as a recovery factor.

Foregoing hypothetical assumptions on "low-dose effects" in terms "radiation embrittlement contains oscillatory component" and "radiation annealing of the radiation embrittlement" is questionable and needs additional experimental verification and profound scientific study.

The information gained would be relevant to the RPV degradation mechanisms consideration and understanding, also to possible current PWR generation lifetime extension evaluation.

\section{References}

[1] E. Krasikov and V. Nikolaenko, Dose rate effect on WWER-440/213 RPV materials embrittlement, Atomic Energy 97 (2004), 177-182 (in Russian).

[2] A. Fabry and R. Gerard, In-service embrittlement of the pressure vessel welds at the Doel I and II NPPs, in D. Gelles and R. Nanstad (Eds.), Effects of radiation on materials ASTM STP 1270, West Conshohocken, PA, Ann Arbor, (1996), 294-319.

[3] T. U. Marston, Radiation embrittlement significance of its effects on integrity and operation of PWR pressure vessels, Nuclear Safety 21 (1980), 724-730.

[4] K. L. Murty, Is neutron irradiation exposure always detrimental to metals (steels)? Nature 308 (1984), 51-52.

[5] H. Viehrig and M. Valo, Fracture mechanics characterization of the beltline welding seam of the decommissioned WWER-440 RPV of NPP Greifswald Unit 4, International Journal of Pressure Vessels and Piping 89 (2012), 129-136. 